\title{
Saccade Dysmetria during Functional Perturbation of the Caudal Fastigial Nucleus in the Monkey
}

\author{
LAURENT GOFFART, ${ }^{a}$ LONGTANG L. CHEN, ${ }^{b}$ AND DAVID L. SPARKS ${ }^{b}$ \\ ${ }^{a}$ INSERM U534, Bron, France \\ ${ }^{b}$ Division of Neuroscience, Baylor College of Medicine, Houston, Texas 77030, USA
}

\begin{abstract}
The caudal fastigial nucleus (cFN) is the output nucleus by which the medioposterior cerebellum influences the brainstem saccade generator. In the monkey, inactivation of one cFN by local injection of muscimol impairs all saccades: ipsiversive saccades become hypermetric, contraversive saccades become hypometric, and saccades aimed at a target located in the upper or lower visual fields are biased horizontally toward the injected side. The pharmacological action of muscimol does not allow deficits that are presaccadic to be distinguished from those occurring during saccade execution. To determine the interval during which altered cFN activity affects saccade accuracy, we applied low-frequency electrical microstimulation $(100 \mathrm{~Hz}$ for $100-300 \mathrm{~ms})$ to the $\mathrm{cFN}$ of three monkeys while they were making saccades toward a flashed target. Similar to the effect of muscimol injection in cFN, low-frequency microstimulation biased all saccades toward the ipsilateral side. When the microstimulation was applied after target flash and before saccade onset, the ipsilateral bias was absent. However, when the stimulation was applied during the ongoing movement, the saccade trajectory was biased toward the stimulated side. The muscimol-like effect of the microstimulation suggests that the stimulation inhibits cFN activity, possibly by recruiting the inhibitory afferents from the cerebellar vermis (axons of Purkinje cells). Low-frequency microstimulation had to be applied during the saccade to bias its trajectory. These data suggest that the ipsilateral horizontal bias observed during muscimol inactivation results from an imbalance in the intrasaccadic activity between the two caudal fastigial nuclei.
\end{abstract}

KEYWORDS: saccades; cerebellum; fastigial; reversible inactivation; microstimulation; monkey

\section{INTRODUCTION}

The medioposterior cerebellum is one of the most important brain regions involved in the transformation of target-related visual signals into motor commands that move the line of sight accurately toward the target location. Indeed, damage of this cerebellar region severely impairs the accuracy of goal-directed gaze shifts (for

Address for correspondence: Laurent Goffart, Ph.D., INSERM U534, 16 avenue Doyen Lépine, 69500 Bron, France. Voice: 334729134 01; fax: 33472913403.

goffart@lyon.inserm.fr

Ann. N.Y. Acad. Sci. 1004: 220-228 (2003). @ 2003 New York Academy of Sciences. doi: 10.1196/annals.1303.019 
review, see Robinson and Fuchs ${ }^{1}$ and Pélisson and colleagues ${ }^{2}$ ). The medioposterior cerebellum consists of the lobules VIc-VII in the vermis and of their two output nuclei: the caudal fastigial nuclei.

In the head-restrained monkey, the unilateral disinhibition of neuronal activity in the caudal fastigial nucleus (cFN) by local injection of bicuculline ${ }^{3}$ or by lesion of the vermal lobules VI-VII ${ }^{4}$ impairs the accuracy of visually triggered saccades. Saccades toward the disinhibited side (ipsiversive saccades) become hypometric, and saccades toward the opposite side (contraversive saccades) become hypermetric. Conversely, the inactivation of the $\mathrm{cFN}$ by local injection of muscimol leads to hypermetric ipsiversive saccades and hypometric contraversive saccades. ${ }^{5-7}$ In the head-unrestrained cat, after unilateral injection of muscimol in the cFN, ipsiversive combined eye-head gaze shifts become hypermetric, and contraversive ones become hypometric, ${ }^{8,9}$ without change in the contribution of the head to the overall gaze displacement. ${ }^{10}$

Although most $\mathrm{cFN}$ neurons show a steady firing rate during intersaccadic intervals, the dysmetria observed during muscimol injection in the $\mathrm{cFN}$ has been proposed to result from the suppression of saccade-related bursts of activity in cFN. ${ }^{11-13}$ According to this hypothesis, the hypermetria of ipsiversive saccades would be due to the suppression of the burst generated during the late period of the saccades, whereas the hypometria of contraversive saccades would be due to the suppression of the burst that precedes the onset of saccades.

An alternative hypothesis, based on several observations made in the head-unrestrained cat, has been proposed to account for the dysmetria resulting from unilateral muscimol injections into cFN. According to this hypothesis, the dysmetria results from an impaired specification of the movement metrics prior to movement onset, rather than an intrasaccadic deficit. Indeed, the observation of misdirected and inappropriately initiated ipsiversive gaze shifts suggests more than a deficit in the control of movement deceleration. ${ }^{89}$ All these ipsiversive gaze shifts overshoot the target, producing a constant horizontal error: the endpoints are horizontally shifted with respect to the target position, irrespective of the starting gaze position. Moreover, no consistent modifications in the dynamics of the eye, head, and gaze displacements or in the eye-head coupling are observed. Occasional muscimol-induced modifications in dynamics are unrelated to the magnitude of the dysmetria. ${ }^{10}$ Finally, changes in the latency of the gaze and head displacements during cFN inactivation ${ }^{14}$ or after ablation in the oculomotor vermis ${ }^{4}$ support the idea of a deficit unfolding prior to movement onset.

Some observations in the head-restrained monkey suggest that muscimol injection in the $\mathrm{cFN}$ can lead to deficits in the primate that are similar to those observed in the cat. In particular, saccades initiated from various eccentric eye positions and aimed at a visual target located straight ahead all end at approximately the same final position, one that is horizontally shifted relative to the target. This is very reminiscent of the constant horizontal error observed in the cat (see Figure 2H in the article by Ohtsuka and colleagues ${ }^{6}$ ).

\section{PERTURBING THE CAUDAL FASTIGIAL NUCLEUS BY LOCAL INJECTION OF MUSCIMOL}

The effects of inactivating the saccade-related area in the $\mathrm{cFN}$ was studied in three head-restrained monkeys to determine if saccades initiated from various start- 


\section{Control Muscimol (left cFN)}
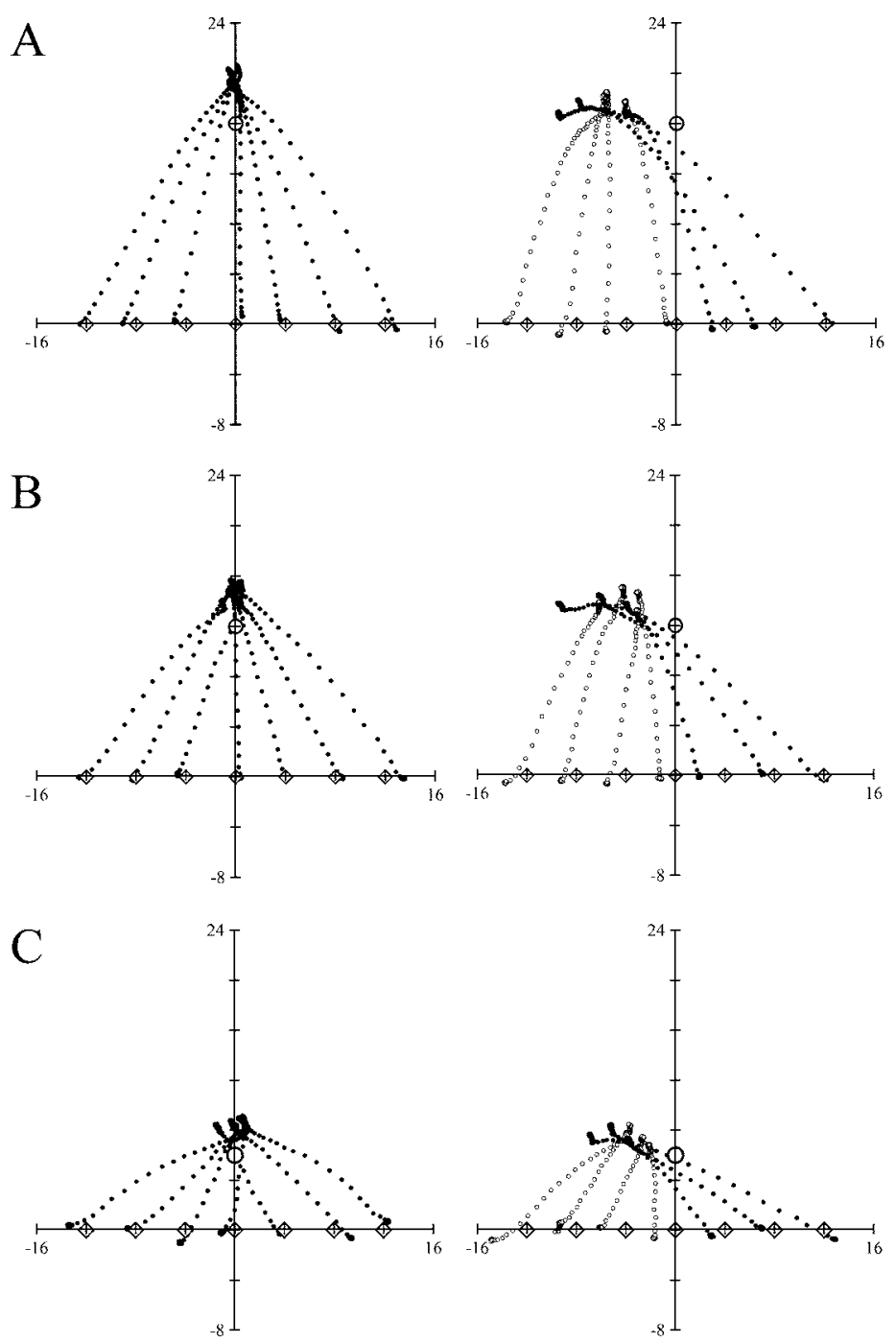

FIGURE 1. Effects on saccade direction and amplitude of perturbation of the $\mathrm{cFN}$ by local injection of muscimol. The saccades were aimed at flashed (duration $=100 \mathrm{~ms}$ ) target LEDs (circles) located (A) $16^{\circ}$, (B) $12^{\circ}$, or $(\mathbf{C}) 6^{\circ}$ on the vertical meridian. Variation in the starting position of the eyes was obtained by using seven different fixation LEDs (diamonds): $\pm 12^{\circ}, \pm 8^{\circ}, \pm 4^{\circ}$, and $0^{\circ}$ along the horizontal meridian. For clarity, saccades starting from fixation LEDs located to the left are plotted with a different symbol (open) from those starting from fixation LEDs located to the right (filled). Volume injected: $0.8 \mu \mathrm{L}(1 \mu \mathrm{g} / \mu \mathrm{L})$. 
ing positions and aimed at the same visual target missed the target with a similar constant horizontal error. FIGURE 1 shows the effect of injecting a small volume of muscimol in the $\mathrm{cFN}$ on the trajectories of representative saccades initiated from various starting positions along the horizontal meridian and aimed at a brief target lightemitting diode $(\mathrm{LED})$ (duration $=100 \mathrm{~ms})$. Target LEDs (circles) were located $16^{\circ}$ $(\mathrm{A}), 12^{\circ}(\mathrm{B})$, or $6^{\circ}(\mathrm{C})$ on the vertical meridian (FIG. 1A-C, respectively). The left panel shows the fixation and the saccade performance before the injection (control). After muscimol injection in the left cFN (right panel), a leftward offset in starting eye position was observed when the animal was viewing the fixation LEDs. This fixation offset was to the right following the injection in the right cFN (results not shown, but see Robinson and colleagues ${ }^{5}$ and Goffart and Pélisson ${ }^{9}$ ). With respect to saccades toward the $16^{\circ}$ upward target (FIG. 1A), the horizontal component of leftward (ipsiversive) saccades was too large, whereas the horizontal component of rightward (contraversive) saccades was too short to acquire the target. Moreover, after the muscimol injection, the initial direction of saccades starting from the straightahead fixation LED was to the left, and saccades in this direction increased, rather than decreased, the horizontal distance between gaze and target positions. Saccades initiated from the $4^{\circ}$ leftward fixation LED had no rightward component even though the target for these trials was located more than $4^{\circ}$ to the right. Saccades starting from fixation LEDs located further to the left had less of a rightward initial direction than would be required for target acquisition. The amplitude of their horizontal component was hypometric. The initial direction of saccades starting from fixation LEDs located to the right had more of a leftward component than that required to look to the target. The amplitude of the horizontal component was hypermetric with an overshoot that increased with more eccentric starting position (or with larger horizontal target eccentricity). Similar effects upon the initial direction were apparent for saccades directed to the $12^{\circ}$ and $6^{\circ}$ targets (FIG. 1B, C, respectively). However, when the vertical eccentricity of the target was reduced, the horizontal error at the end of the saccades was also significantly reduced $\left(-3.9 \pm 0.7,-2.9 \pm 0.7\right.$, and $-1.9 \pm 0.5^{\circ}$ for targets $16^{\circ}, 12^{\circ}$, and $6^{\circ}$ upward, respectively; Mann-Whitney $U$ test, $P<0.01$ for each comparison). The endpoints of saccades starting from the straight-ahead fixation LED were offset relative to the target with a magnitude that increased with the vertical target eccentricity (see also Iwamoto ${ }^{7}$ ). Moreover, when the saccades initiated from the most eccentric fixation LEDs to the left $\left(-8^{\circ}\right.$ or $\left.-12^{\circ}\right)$ were examined, the amplitudes of their horizontal components were larger when the vertical target position decreased from $16^{\circ}$ to $6^{\circ}$ upward.

In summary, saccades initiated from various starting positions did not end at the same final eye position that was shifted relative to the target location by a constant horizontal error. Rather, ipsiversive saccades missed the target with a horizontal error that increased as the eccentric starting deviation of the eyes in the orbit increased and, concomitantly, as horizontal target eccentricity and saccade duration increased. Statistical analysis indicates that the horizontal error of oblique ipsiversive saccades aimed at target with similar eccentricity (for example, $12^{\circ}$ leftward and upward) was not statistically different between saccades initiated from the $12^{\circ}$ eccentric fixation LED and saccades initiated from the central fixation LED (Mann-Whitney $U$ test, $P$ level $>0.10$ ). Contraversive saccades toward one given target seemed to reach the same final eye position, but the location of saccade endpoints was different for different targets. 
A

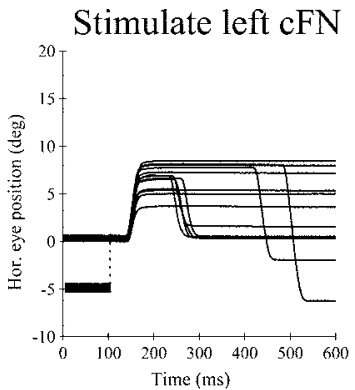

$\mathrm{B}$

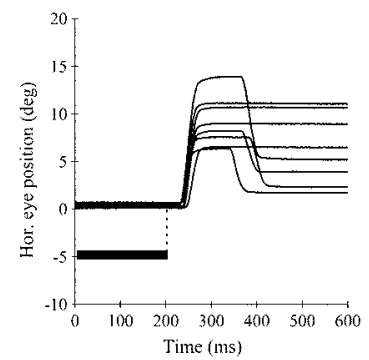

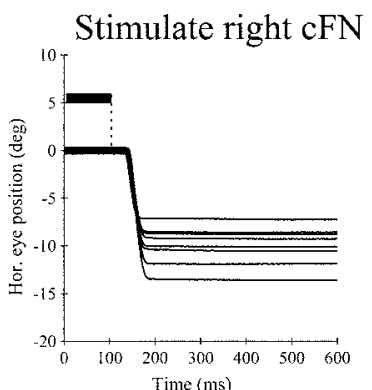

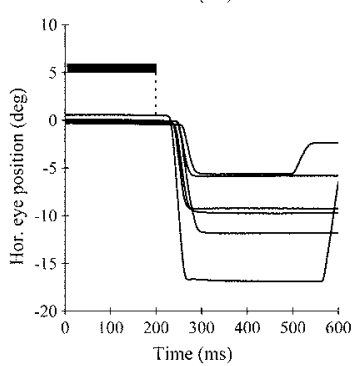

FIGURE 2. Electrical microstimulation of the dorsocaudal portion of the fastigial nucleus. The stimulation train (black horizontal bar; 100-Hz, 0.2-ms pulse; duration $=100 \mathrm{~ms}$ in $\mathbf{A}, 200 \mathrm{~ms}$ in $\mathbf{B}$ ) was applied while the animal was waiting for the appearance of a visual target. Current intensity was $35 \mu \mathrm{A}$ and $20 \mu \mathrm{A}$ for the left $\mathrm{cFN}$ and right $\mathrm{cFN}$ of the same animal, respectively.

\section{PERTURBING THE CAUDAL FASTIGIAL NUCLEUS BY LOW-FREQUENCY ELECTRICAL MICROSTIMULATION}

From the inactivation data, it is impossible to determine whether the dysmetria is due to presaccadic or intrasaccadic imbalances in the activity of the two cFNs. Thus, we developed a technique that allowed more transient inhibition of the activity of one $\mathrm{cFN}$. This technique relies on the assumption that electrical microstimulation of the dorsocaudal portion of the fastigial nucleus inhibits $\mathrm{cFN}$ neurons by activating the inhibitory axons of Purkinje cells from the vermis. ${ }^{15}$ By manipulating the duration of the microstimulation and its delay with respect to the target presentation, we were able to determine the critical period during which cFN activity influences saccade accuracy within the time interval between the onset of the target and the end of the saccade.

FIGURE 2 illustrates the effect of microstimulating the dorsocaudal portion of the left and right fastigial nucleus with a $100-\mathrm{Hz}$ train during a gap interval $(500 \mathrm{~ms})$ when the animal was waiting for the appearance of a visual target. Eye position was stable during the entire period of microstimulation (black horizontal bar; duration = $100 \mathrm{~ms}$ in FIG. 2A, $200 \mathrm{~ms}$ in FIG. 2B), but a "rebound" saccade was generated 40 to $60 \mathrm{~ms}$ after the offset of the microstimulation train. This rebound saccade was always directed toward the side contralateral to the stimulated side, that is, rightward 

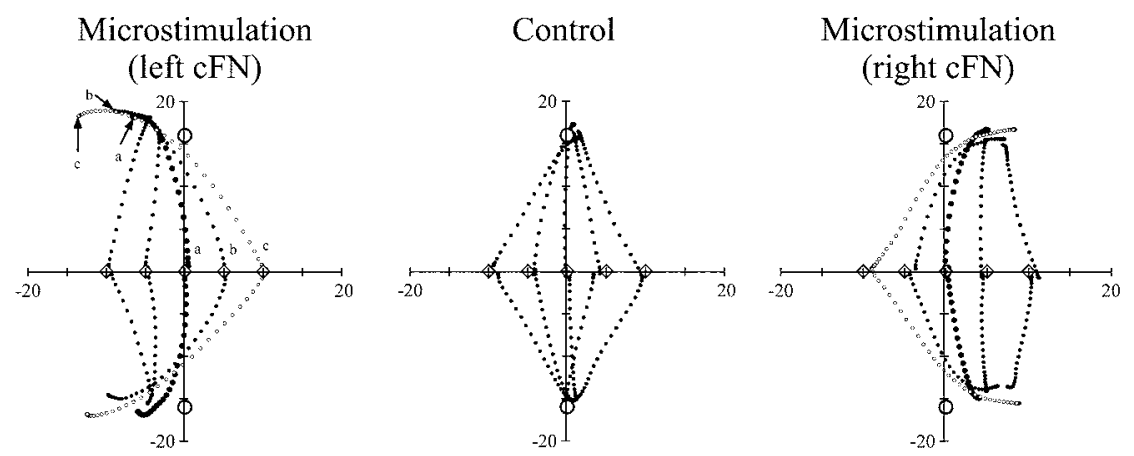

FIGURE 3. Effects on saccade direction and amplitude produced by microstimulation of the cFN. Saccades were directed toward a brief target (circles; duration $=100 \mathrm{~ms}$ ) presented $16^{\circ}$ above or below the central fixation LED. The stimulation train was synchronized with respect to the target offset. It overlapped the interval during which the line of sight was moving toward the target. Variations in the horizontal starting position of the eyes were obtained by using five different fixation LEDs (diamonds): $\pm 10^{\circ} ; \pm 5^{\circ}$, and $0^{\circ}$ along the horizontal meridian. Stimulation parameters for left cFN (same site as in FIG. 2, left panels): 100 $\mathrm{Hz}, 100$ or $200 \mathrm{~ms}, 20 \mu \mathrm{A}$; stimulation parameters for right cFN: $100 \mathrm{~Hz}, 300 \mathrm{~ms}, 100 \mu \mathrm{A}$.

when the left cFN was stimulated (left panels in FIG. 2), and leftward when the right cFN was stimulated (right panels in FIG. 2). Previous work has shown that contralateral saccades are also evoked by microstimulating the axons of cFN neurons. ${ }^{15}$ The contralateral saccades illustrated in FIGURE 2 could thus result from the rebound depolarization and spike bursting by $\mathrm{cFN}$ neurons following a prolonged microstimulation-induced hyperpolarization. ${ }^{16}$ When applied while the monkey was preparing a goal-directed saccade, the microstimulation train had an effect on saccade trajectory that did indeed suggest that the microstimulation inhibits the activity in the cFN.

FIGURE 3 shows the effect of a microstimulation train at $100 \mathrm{~Hz}$ on the trajectory of saccades initiated from different horizontal starting positions and aimed at a brief target (duration $=100 \mathrm{~ms}$ ) presented either $16^{\circ}$ upward or downward. The stimulation was synchronized with target offset. The rebound saccades were generated after the saccades shown (and elicited after stimulation offset) and removed for clarity. Like the muscimol injection in the cFN (see FIG. 1), the trajectory of saccades was biased toward the perturbed side; the horizontal component of all saccades was impaired without change in the vertical component. The horizontal component of ipsiversive saccades was hypermetric, whereas for contraversive saccades it was hypometric. The greater the target eccentricity relative to the starting eye position, the larger the hypermetria of ipsiversive saccades. The muscimol-like effect of the microstimulation on visually triggered saccades strongly suggests that the stimulation inhibits the activity of $\mathrm{cFN}$ neurons by recruiting the inhibitory influence of Purkinje cells axons innervating the nucleus, a conclusion that is in accordance with conclusions reached by Noda's group. ${ }^{15}$

FIGURE 4 shows the effect of varying the interval during which the microstimulation was applied upon the trajectory and the accuracy of saccades. In the trials 

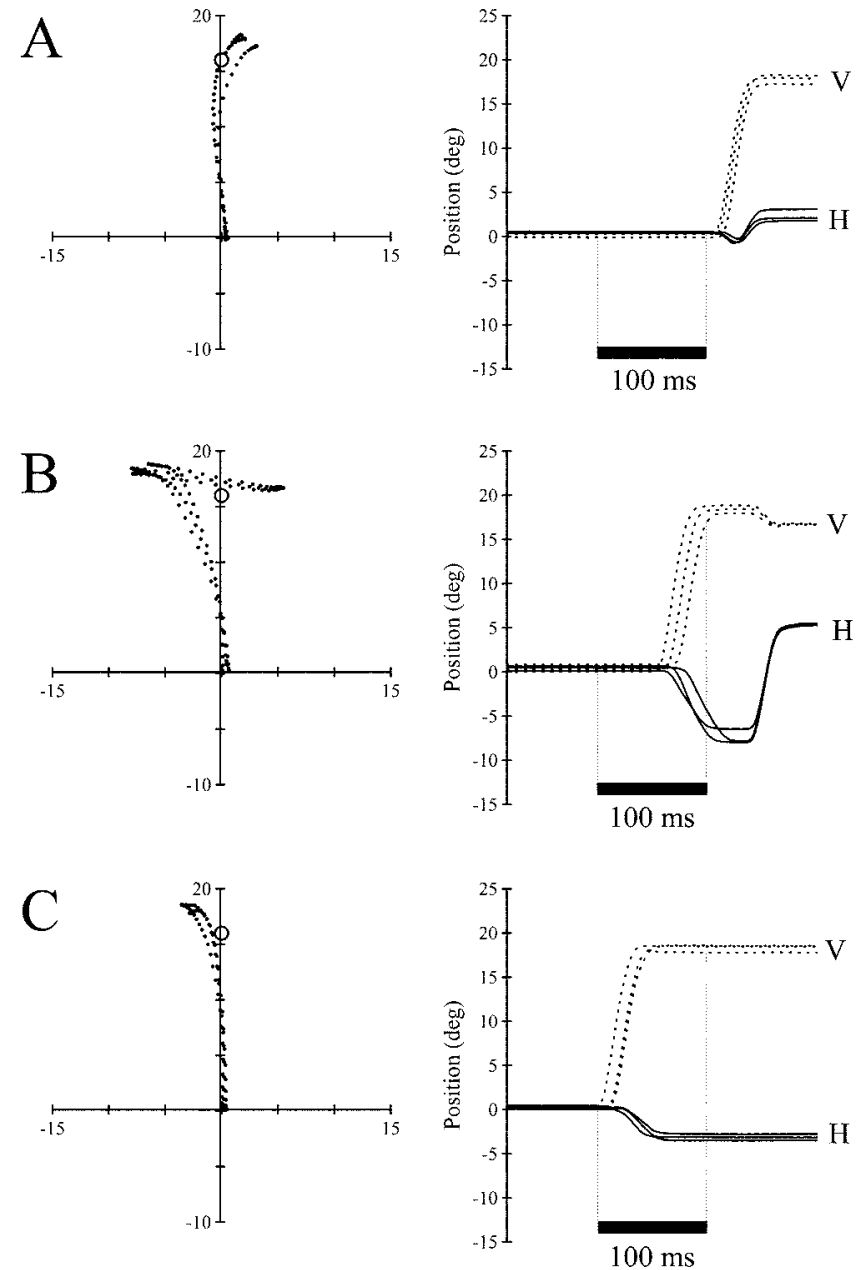

FIGURE 4. Effects of varying the period of stimulation within the target-offset/ saccade-end interval. The fixation LED was located straight ahead, and the target LED was flashed $16^{\circ}$ above the fixation stimulus. The microstimulation (black horizontal bar; 100$\mathrm{Hz}, 0.2$-ms pulse; 100-ms duration; $45 \mu \mathrm{A}$ ) was applied (A) immediately, (B) $50 \mathrm{~ms}$, or (C) or $100 \mathrm{~ms}$ after the target LED was extinguished. The $x-y$ plots of eye position are shown on the left, and plots of horizontal $(\mathrm{H})$ and vertical $(\mathrm{V})$ eye positions as a function of time are shown on the right. The black horizontal bar represents the period when microstimulation was delivered. The traces are aligned on the onset of the microstimulation train. 
shown as examples, the fixation LED was located straight ahead, and the target LED was flashed $16^{\circ}$ above the fixation LED. The microstimulation was applied immediately, $50 \mathrm{~ms}$, or $100 \mathrm{~ms}$ (FIG. 4A-C, respectively) after the target LED was extinguished. The stimulation duration was set to $100 \mathrm{~ms}$ so that on some trials it could be restricted to the period prior to saccade initiation. In that particular case (FIG. 4A), only small effects on vertical saccades were observed: a slight final deviation away from the stimulation side occurred. When the microstimulation was applied during the overall saccade period (FIG. 4B), a much larger leftward bias was observed in the trajectory. A rightward rebound saccade immediately followed the stimulation offset. The ipsilateral bias in saccade trajectory was less important when the microstimulation was applied slightly before saccade onset (FIG. 4C). The figure also shows that the amount of microstimulation (duration) applied before (up to $10 \mathrm{~ms}$ before) the saccade was launched toward the visual target is not critical in determining the amount of deviation in saccade trajectory.

\section{CONCLUSION}

Low-frequency microstimulation of the dorsocaudal portion of the fastigial nucleus biases the trajectory of saccades toward the stimulated side. This effect is similar to the effect of muscimol injection in the cFN. The microstimulation must be applied during the saccade to bias its trajectory (see also Keller and colleagues ${ }^{17}$ ). More experiments using a larger range of intervals between stimulation onset and saccade onset are needed. But when an efferent delay of approximately $10 \mathrm{~ms}$ is taken into account, our data suggest that it is only the microstimulation immediately before saccade onset and during the execution period that is effective in modifying the trajectory of a saccade.

Further experiments are required to verify that these conclusions also hold in the head-unrestrained monkey. If so, the particular dysmetria observed in the headunrestrained cat ${ }^{9}$ may be due to the nature of the behavioral responses evoked by the target. Indeed, in the cat experiments, the food target was triggering an orienting gaze shift but also an orienting movement of the mouth (and thus of the head). In the monkey experiments, target acquisition required only a shift of the line of sight.

\section{ACKNOWLEDGMENTS}

We thank Kathy Pearson for invaluable programming assistance. This work was supported by the National Institutes of Health (EY02520, EY01189 to D.L.S. and EY13444 to L.L.C.); the Human Frontier Science Program (LT 59/96); and the Centre National de la Recherche Scientifique (to L.G.).

\section{REFERENCES}

1. Robinson, F.R. \& A.F. FuCHS. 2001. The role of the cerebellum in voluntary eye movements. Annu. Rev. Neurosci. 24: 981-1004.

2. PÉlisson, D., L. Goffart \& A. Guillaume. 2003. Control of saccadic eye movements and combined eye/head gaze shifts by the medio-posterior cerebellum. Prog. Brain Res. 142: 69-89. 
3. SATO, H. \& H. NodA. 1992. Saccadic dysmetria induced by transient functional decortication of the cerebellar vermis. Exp. Brain Res. 88: 455-458.

4. TAKAGI, M., D.S. ZEE \& R.J. TAMARGO. 1998. Effects of lesions of the oculomotor vermis on eye movements in primate: saccades. J. Neurophysiol. 80: 1911-1931.

5. Robinson, F.R., A. StRAubE \& A.F. FuCHS. 1993. Role of the caudal fastigial nucleus in saccade generation. II. Effects of muscimol inactivation. J. Neurophysiol. 70: $1741-1758$.

6. OhtsukA, K., H. SATO \& H. NoDA. 1994. Saccadic burst neurons in the fastigial nucleus are not involved in compensating for orbital nonlinearities. J. Neurophysiol. 71: $1976-1980$.

7. IwAMOTO, Y. 2002. Saccadic dysmetria following inactivation of the primate fastigial oculomotor region. Neurosci. Lett. 325: 211-215.

8. GofFarT, L. \& D. PÉlisson. 1994. Cerebellar contribution to the spatial encoding of orienting gaze shifts in the head-free cat. J. Neurophysiol. 72: 2547-2550.

9. GOFFART, L. \& D. PÉLISSON. 1998. Orienting gaze shifts during muscimol inactivation of caudal fastigial nucleus in the cat. I. Gaze dysmetria. J. Neurophysiol. 79: 19421958.

10. Goffart, L., D. Pélisson \& A. Guillaume. 1998. Orienting gaze shifts during muscimol inactivation of caudal fastigial nucleus in the cat. II. Dynamics and eye-head coupling. J. Neurophysiol. 79: 1959-1976.

11. OHTSUKA, K. \& H. NoDA. 1991. Saccadic burst neurons in the oculomotor region of the fastigial nucleus of macaque monkeys. J. Neurophysiol. 65: 1422-1434.

12. FuCHS, A.F., F.R. RoBINSON \& A. STRAUBE. 1993. Role of the caudal fastigial nucleus in saccade generation. I. Neuronal discharge patterns. J. Neurophysiol. 70: 17121740 .

13. Helmchen, C., A. Straube \& U. Büttner. 1994. Saccade-related activity in the fastigial oculomotor region of the macaque monkey during spontaneous eye movements in light and darkness. Exp. Brain Res. 98: 474-482.

14. GOFFART, L. \& D. PÉLISSON. 1997. Changes in initiation of orienting gaze shifts after muscimol inactivation of the caudal fastigial nucleus in the cat. J. Physiol. (Lond.) 503: 657-671.

15. NODA, H., S. MuraKami, J. YAMADA, et al. 1988. Saccadic eye movements evoked by microstimulation of the fastigial nucleus of macaque monkeys. J. Neurophysiol. 60: 1036-1052.

16. AizENMAN, C.D. \& D.J. LinDEN. 1999. Regulation of rebound depolarization and spontaneous firing patterns of deep nuclear neurons in slices of rat cerebellum. J. Neurophysiol. 82: 1697-1709.

17. Keller, E.L., D.P. Slakey \& W.F. CRAndall. 1983. Microstimulation of the primate cerebellar vermis during saccadic eye movements. Brain Res. 288: 131-143. 